\title{
Volichenko Algebras as Algebras of Differential Operators
}

\author{
Uma N IYER \\ Department of Mathematics, SUNY Potsdam, NY 13676, USA \\ E-mail: iyerun@potsdam.edu
}

Received February 7, 2005; Accepted in Revised Form June 8, 2005

\section{Introduction}

Throughout this paper, $\mathbb{k}$ denotes a field of characteristic 0 and all tensor products are over $\mathbb{k}$. Further, $\mathbb{k}[X ; n]$ is the polynomial algebra in $n$ commuting indeterminates $X=$ $\left(x_{1}, x_{2}, \cdots, x_{n}\right)$ and $\Lambda[Y ; m]$ is the Grassmann algebra in $n$ anti-commuting indeterminates $Y=\left(y_{1}, y_{2}, \cdots, y_{m}\right)$.

Supersymmetries are symmetries of supervarieties, i.e., objects, functions on which depend on both usual commuting (even) variables and on anticommuting (odd) ones. For numerous applications of supersymmetry and for basics, see [3], [2] and [10]. Supersymmetries widened the notion of group in order to be able to mix Bose and Fermi particles. However, the collection of morphisms of supervarieties (locally, of its superalgebra of functions $F$ ) - supersymmetries - is not the largest possible group of automorphisms of the algebra $F$, with superstructure ignored. Besides, not every subalgebra or a quotient of a supercommutative superalgebra is supercommutative, whereas they are metaabelian and the notion of superscheme was first given ([7]) in terms of such, not necessarily homogeneous, subalgebras and quotients of supercommutative superalgebras. Recall that a ring $M$ is said to be metaabelian if $[a,[b, c]]=0$ for all $a, b, c \in M$, where $[a, b]=a b-b a$. Volichenko showed (see [8]) that every metaabelian algebra can be realized as a nonhomogeneous subalgebra of a universal supercommutative superalgebra, called its supercommutative envelope.

The purpose of this note it to construct an appropriate analog of differential operators on metaabelian algebras, more precisely, viewing a metaabelian algebra $M$ as an analog of the algebra of functions, construct the corresponding algebra of vector fields.

Lunts and Rosenberg ([9]) constructed algebras of differential operators on (graded) noncommutative algebras. In particular, one can study differential operators on superalgebras. Superderivations of a superalgebra, which are first order differential operators, form a Lie superalgebra.

In a work aborted by his death, Volichenko gave a conjectural intrinsic description of nongraded subalgebras of Lie superalgebras. In his memory then, Leites and Serganova ([8]) called such subalgebras Volichenko algebras and (under a technical assumption) listed simple Volichenko algebras (finite dimensional and of vector fields). Like the list of simple 
Lie superalgebras about doubles the list of simple Lie algebras of the same type [6], the list of simple Volichenko algebras about doubles the list of simple Lie superalgebras.

We construct differential operators on metaabelian algebras so that certain first order differential operators form a Volichenko algebra.

Preliminaries on differential operators defined by Lunts and Rosenberg are covered in section 2 followed by a section introducing superalgebras. In section 4 , we study different kinds of differential operators on metaabelian algebras. Here, (4.1.2) we also construct a special tensor product of two metaabelian algebras. Note that the usual tensor product of metaabelian algebras is not necessarily metaabelian.

Given two metaabelian algebras $M$ and $N$, let $S_{M}$ and $S_{N}$ be their respective supercommutative envelopes. Then, their metaabelian tensor product, $M \stackrel{m}{\otimes} N$ is defined as a natural nonhomogeneous subalgebra of $S_{M} \stackrel{s}{\otimes} S_{N}$. Molotkov has already defined a special tensor product of two metaabelian alebras (see appendix in [8]). Our constructions are different but we arrive at isomorphic metaabelian algebras because of the universal property (Proposition 4.1.3.)

Using the machinery of super differential operators on superalgebras, we define the Volichenko differential operators $V D$ in section 4.2. That is, let $S_{M}$ denote the supercommutative envelope of a metaabelian algebra $M$, along with a natural odd derivation $d$ on $S_{M}$ given by Volichenko. We let $D_{\beta}\left(S_{M}\right)$ denote the algebra of superdifferential (this is a specialization for the super case of the $\beta$-differential introduced in [9]) operators on $S_{M}$. Then,

$$
V D(M)=\left\{\varphi_{0}+\left[d, \varphi_{0}\right] \mid \varphi_{0} \in D_{\beta}(S) \text { is even }\right\} .
$$

It then follows (Proposition 4.2.1) that $V D(M)=\cup_{n \geq 0} V D^{n}(M)$ is a filtered algebra with $V D^{0}(M)$ being the subalgebra of left multiplication homomorphisms in $M$ and $V D^{1}(M)$ the direct sum as vector spaces of $V D^{0}(M)$ and a Volichenko algebra. We also show that there is a natural homomorphism $V D(M) \stackrel{m}{\otimes} V D(N) \longrightarrow V D(M \stackrel{m}{\otimes} N)$ for $M, N$ metaabelian algebras.

Finally, in section 4.2.3, we describe the Volichenko differential operators on the metaabelian algebra $\mathbb{k}[Z]$, the subalgebra of $\mathbb{k}[X ; n] \otimes \Lambda[Y ; n]$ generated by $Z$, where $z_{i}=x_{i}+y_{i}$.

\section{Preliminaries from [9]}

\section{$2.1 \quad D_{\beta}(R)$, the space of $\beta$-differential operators.}

Let $\Gamma$ be an abelian group. Fix a bicharacter $\beta: \Gamma \times \Gamma \longrightarrow \mathbb{k}^{*}$. Let $R$ be a $\Gamma$-graded $\mathbb{k}$-algebra and $M$ a $\Gamma$-graded $R$-bimodule. Let $Z_{\beta}(M)$ denote the $\beta$-center of $M$ defined as the $\mathbb{k}$-span of homogeneous elements $m \in M$ such that

$$
m r=\beta\left(d_{m}, d_{r}\right) r m \text { for any homogeneous } r \in R,
$$

where $d_{x}$ denotes the degree of $x$. The $\beta$-differential part of $M$ is defined as follows: Let $M_{0}$ be the $R$-bimodule generated by $Z_{\beta}(M)$. Having defined $M_{i}$, define $M_{i+1}$ as the $R$-bimodule generated by the homogeneous $m \in M$ such that

$$
m r-\beta\left(d_{m}, d_{r}\right) r m \in M_{i} \text { for any homogeneous } r \in R .
$$


Each $M_{i}$ is called the $i$ th $\beta$-differential part of $M$. The $\beta$-differential part of $M$ is the filtered, $\Gamma$-graded $R$-bimodule

$$
M_{\beta}=\cup_{i \geq 0} M_{\beta i}
$$

The $\beta$-differential operators on $R$ are the $\beta$-differential parts of the elements of the $R$-bimodule $M=\operatorname{grHom}_{\mathbb{k}}(R, R)$. The $R$-bimodule structure on $M$ is as follows:

$$
(r \varphi s)(t)=r \varphi(s t) \quad \text { for any } \quad r, s, t \in R, \varphi \in \operatorname{grHom}_{\mathbb{k}}(R, R) .
$$

The $R$-bimodule of $\beta$-differential operators is denoted by $D_{\beta}(R)$; the $i$ th $\beta$-differential part of $\operatorname{grHom}_{\mathbb{k}}(R, R)$ is denoted by $D_{\beta}^{i}(R)$ and the elements in $D_{\beta}^{i}(R)$ are called $\beta$-differential operators of order $i$. One can see that

$$
D_{\beta}^{i}(R) D_{\beta}^{j}(R) \subset D_{\beta}^{i+j}(R)
$$

Thus, $D_{\beta}(R)$ is a $\mathbb{k}$-algebra.

Remark 2.1.1. Let $\Gamma_{1}$ and $\Gamma_{2}$ be two abelian groups, equipped with their respective bicharacters $\beta_{i}: \Gamma_{i} \times \Gamma_{i} \longrightarrow \mathbb{k}$ for $i=1,2$. Let $R$ and $S$ be two finitely generated $\mathbb{k}$ algebras which are $\Gamma_{1^{-}}$and $\Gamma_{2}$-graded, respectively, such that elements of $\mathbb{k}$ are of degree 0 . Then the usual tensor product $R \otimes S$ is a $\Gamma_{1} \times \Gamma_{2}$-graded $\mathbb{k}$-algebra. Similar to the proof of Theorem 3.1.1 of [4], we can show that $D_{\beta_{1}}(R) \otimes D_{\beta_{2}}(S) \cong D_{\beta_{1} \beta_{2}}(R \otimes S)$ as $R \otimes S$-bimodules and as $\mathbb{k}$-algebras with the bicharacter given by

$$
\begin{aligned}
& \beta_{1} \beta_{2}:\left(\Gamma_{1} \times \Gamma_{2}\right) \times\left(\Gamma_{1} \times \Gamma_{2}\right) \longrightarrow \mathbb{k}^{*}, \\
& \beta_{1} \beta_{2}((a, b),(c, d))=\beta_{1}(a, c) \beta_{2}(b, d) \text { for any } a, c \in \Gamma_{1} ; b, d \in \Gamma_{2} .
\end{aligned}
$$

\section{$2.2 D_{q}(R)$, the space of quantum differential operators.}

These operators are defined for the same set-up as before, i.e., $\beta: \Gamma \times \Gamma \longrightarrow \mathbb{k}^{*}$ is a fixed bicharacter.

Let $R$ be a $\Gamma$-graded $\mathbb{k}$-algebra and $M$ be a $\Gamma$-graded $R$-bimodule. Denoted by $Z_{q}(M)$ the $q$-center of $M$ defined as the $\mathbb{k}$-span of homogeneous elements $m \in M$ for which there exists a $d \in \Gamma$ such that

$$
m r=\beta\left(d, d_{r}\right) r m \text { for any homogeneous } r \in R .
$$

Note that $d$ depends on $m$, but is not necessarily the degree of $m$, and neither does it have to be unique. The rest of the construction follows as before for $\beta$-differential operators. We denote by $M_{q}=\cup_{i \geq 0} M_{q i}$ the q-differential part of $M$, where $M_{q i}$ denotes the $i$ th $q$-differential part. Likewise, $D_{q}(R)=\cup_{i \geq 0} D_{q}^{i}(R)$ is the ring of $q$-differential operators, where $D_{q}^{i}(R)$ is the $R$-bimodule of $q$-differential operators of order $i$ on $R$. For each $a \in \Gamma$, we define the grading map $\sigma_{a} \in D_{q}^{0}(R)$ by setting

$$
\sigma_{a}(r)=\beta\left(a, d_{r}\right) r
$$


Remark 2.2.1. (1) In the section covering preliminaries in [5], it is shown that $D_{q}^{0}(R)$ is a $\Gamma$-graded $\mathbb{k}$-algebra generated by homomorphisms given by left multiplications, right multiplications, and grading (Lemma 1.2.1 of [5]). The $R$-bimodule $D_{q}^{n+1}(R)$ is generated over $D_{q}^{0}(R)$ by the $\mathbb{k}$-span of homogeneous $\varphi$ such that $(\varphi r-r \varphi) \in D_{q}^{n}(R)$ (Corollary 1.2.1 of $[5])$.

(2) For the same set-up as that of Remark 2.1.1, we have $D_{q}(R \otimes S) \cong D_{q}(R) \otimes D_{q}(S)$ as $R \otimes S$-bimodules and as $\mathbb{k}$-algebras.

\section{3 $D(R)$, the space of the usual differential operators.}

For a non-graded set-up, in the above definitions, we have to merely consider $R$ as trivially graded. That is, we consider $R=R_{0}, \beta \equiv 1, M$ is an $R$-bimodule, and the ring of differential operators on $R$, denoted by $D(R)$, is the differential part of $\operatorname{Hom}_{\mathbb{k}}(R)$.

Remark 2.3.1. (1) By Theorem 3.1.1 of [4], if $R$ and $S$ are finitely generated $\mathbb{k}$-algebras, then $D(R \otimes S) \cong D(R) \otimes D(S)$ as $R \otimes S$-bimodules and as $\mathbb{k}$-algebras.

(2) If $R$ is a $\Gamma$-graded $\mathbb{k}$-algebra and finitely generated as an algebra by homogeneous elements, then $D(R) \subset \operatorname{grHom}_{\mathbb{k}}(R, R)$ by Theorem 3.2.1 of [4].

For the rest of this article, we fix a few notations: For any $r \in R$, let $\lambda_{r}, \rho_{r} \in \operatorname{Hom}(R, R)$ be defined as

$$
\lambda_{r}(s)=r s \text { and } \rho_{r}(s)=s r \text { for any } s \in R .
$$

For any homomorphism $\varphi \in \operatorname{Hom}(R, R)$ and $x \in R$, we denote $[\varphi, x]:=\left[\varphi, \lambda_{x}\right]$.

\section{Superalgebras}

Let $\mathbb{Z}_{2}:=\mathbb{Z} / 2$. A $\mathbb{Z}_{2}$-graded $\mathbb{k}$-algebra $R=R_{0} \oplus R_{1}$ is called a superalgebra. Elements of $R_{0}$ are called even and those of $R_{1}$ are called odd. For a homogeneous element $a \in R$, we let $p(a)$ denote its parity (which is the same as degree in this case). The ring $R$ is called supercommutative if, for any homogeneous elements $a, b \in R$, we have $a b=(-1)^{p(a) p(b)} b a$. Throughout our paper, the underlying field $\mathbb{k}$ is trivially $\mathbb{Z}_{2}$-graded, i.e., all the scalars are even.

We define a bicharacter $\beta: \mathbb{Z}_{2} \times \mathbb{Z}_{2} \longrightarrow \mathbb{k}^{*}$ by setting $\beta(x, y)=(-1)^{x y}$ and define the $\beta$-differential operators on supercommutative superalgebras as the elements of $\mathbb{k}$-algebra $D_{\beta}(R)$.

Remark 3.0.2. (1) The algebra of 0th order $\beta$-differential operators on a superalgebra $R$ is generated by $\lambda_{r}, \rho_{s}^{\beta}$ for homogeneous $r, s \in R$, where

$$
\rho_{s}^{\beta}(t)=(-1)^{p(t) p(s)} t s .
$$

When $R$ is supercommutative, we have $\rho_{s}^{\beta}=\lambda_{s}$ for any homogeneous $s \in R$.

(2) Let $\varphi$ be a left superderivation on a superalgebra $R$. That is, $\varphi$ is a homogeneous homomorphism such that

$$
\varphi(r s)=\varphi(r) s+(-1)^{p(\varphi) p(r)} r \varphi(s) \text { for } r \text { homogeneous. }
$$


That is,

$$
\varphi r-\beta(p(\varphi), p(r)) r \varphi=\lambda_{\varphi(r)} \in D_{\beta}^{0}(R) .
$$

Hence $\varphi \in D_{\beta}^{1}(R)$, so first order $\beta$-differential operators on a supercommutative superalgebra form a Lie superalgebra. For preliminaries on (Lie) superalgebras, see [6], [2]. Let

$$
[m, n]_{\beta}=m n-(-1)^{p(m) p(n)} n m .
$$

Proposition 3.0.1. Let $R=R_{0} \oplus R_{1}$ be a supercommutative superalgebra. A homogeneous homomorphism $\varphi$ belongs to $D_{\beta}^{n}(R)$ if and only if

$$
\left[\cdots\left[\left[\varphi, a_{1}\right]_{\beta}, a_{2}\right]_{\beta}, \cdots, a_{n+1}\right]_{\beta}=0 \text { for all homogeneous } a_{i} \in R \text {. }
$$

Proof. Let a homogeneous $\varphi \in D_{\beta}^{0}(R)$ be a generator. That is, $[\varphi, a]_{\beta}=0$ for any homogeneous $a \in R$ or $\varphi=\rho_{r}^{\beta}$. But $\rho_{r}^{\beta}=\lambda_{r}$. Thus, the proposition is true for $n=0$. The rest follows by induction.

Corollary 3.0.1. Let $R=R_{0} \oplus R_{1}$ be a supercommutative superalgebra. Then $D_{\beta}^{1}(R)=$ $D_{\beta}^{0}(R) \oplus \operatorname{Der}_{\beta}(R)$ as vector spaces where $\operatorname{Der}_{\beta}(R)$ denotes the left $R$-module of left superderivations on $R$.

Proof. The sum is direct because superderivations vanish at 1 . Given homogeneous $\varphi \in D_{\beta}^{1}(R)$, we let $\psi=\varphi-\rho_{\varphi(1)}^{\beta}$. Clearly, $\psi$ is homogeneous with $\psi(1)=0$, and

$$
[\psi, r]_{\beta}=[\varphi, r]_{\beta} \text { for all } r \in R .
$$

Hence, $\psi \in D_{\beta}^{1}(R)$. Since $\left[\left[\psi, r_{1}\right]_{\beta}, r_{2}\right]_{\beta}=0$, we see that $[\psi, r]_{\beta}=\lambda_{f_{r}}$. Since $\psi(1)=0$, we have $\psi(r)=f_{r}$. In particular, $[\psi, r]=\lambda_{\psi(r)}$, hence $\psi$ is a left superderivation.

Lemma 3.0.1. Let $\left\{R_{\alpha}\right\}$ be a family of supercommutative superalgebras. Let $\prod_{\alpha} R_{\alpha}$ denote the unital supercommutative superalgebra, the direct product of the $R_{\alpha}$. Then, $D_{\beta}\left(\prod_{\alpha} R_{\alpha}\right) \cong \prod_{\alpha} D_{\beta}\left(R_{\alpha}\right)$ as filtered algebras.

Proof. Note, $D_{\beta}^{0}\left(\prod_{\alpha} R_{\alpha}\right) \cong \prod_{\alpha} D_{\beta}^{0}\left(R_{\alpha}\right)$. By induction we show that $D^{n}\left(\prod R_{\alpha}\right) \subset$ $\prod_{\alpha} \operatorname{End}_{\mathbb{k}}\left(R_{\alpha}\right)$ which inturn will imply the result.

Consider a homogeneous

$$
\Phi=\prod_{\alpha_{1}, \alpha_{2}} \varphi_{\alpha_{1}, \alpha_{2}} \in \operatorname{End}_{\mathbb{k}}\left(\prod R_{\alpha}\right)
$$

where $\varphi_{\alpha_{1}, \alpha_{2}} \in \operatorname{Hom}\left(R_{\alpha_{1}}, R_{\alpha_{2}}\right)$. Fix an $a_{\alpha_{0}} \in R_{\alpha_{0}}$. We have

$$
\left[\Phi, a_{\alpha_{0}}\right]_{\beta}=\left[\varphi_{\alpha_{0}, \alpha_{0}}, a_{\alpha_{0}}\right]_{\beta}+\prod_{\alpha_{2} \neq \alpha_{0}} \varphi_{\alpha_{0}, \alpha_{2}} a_{\alpha_{0}}-(-1)^{c} \prod_{\alpha_{1} \neq \alpha_{0}} a_{\alpha_{0}} \varphi_{\alpha_{1}, \alpha_{0}} \in \prod \operatorname{End}_{\mathbb{k}}\left(R_{\alpha}\right)
$$

for some $c \in\{ \pm 1\}$. Hence, $\varphi_{\alpha_{1}, \alpha_{2}} a_{\alpha_{0}}=a_{\alpha_{0}} \varphi_{\alpha_{1}, \alpha_{2}}=0$ for all $a_{\alpha_{0}} \in R_{\alpha_{0}}$. In particular, we have $\varphi_{\alpha_{0}, \alpha_{2}}=0=\varphi_{\alpha_{1}, \alpha_{0}}$ for $\alpha_{1} \neq \alpha_{0}$ and $\alpha_{2} \neq \alpha_{0}$. Hence the result. 


\section{Metaabelian algebras}

Let $R$ be a metaabelian $\mathbb{k}$-algebra. A suitable algebra of differential operators on $R$ is $D(R)$ computed for $R$ viewed as a non-graded algebra.

Proposition 4.0.2. (1) Let $R$ be a metaabelian $\mathbb{k}$-algebra. If $\varphi \in D^{n}(R)$, then

$$
\left[\cdots\left[\left[\varphi, x_{1}\right], x_{2}\right], \cdots, x_{2(n+1)}\right]=0
$$

for all $x_{i} \in R$.

(2) In particular, a homomorphism $\varphi$ belongs to $D(R)$ if and only if

$$
\left[\cdots\left[\left[\varphi, x_{1}\right], x_{2}\right], \cdots, x_{n}\right]=0 \text { for some } n \geq 0 \text { and all } x_{i} \in R .
$$

Proof. By definition of $D(R)$, if $\left[\cdots\left[\left[\varphi, x_{1}\right], x_{2}\right], \cdots, x_{n+1}\right]=0$ for all $x_{i} \in R$, then $\varphi \in$ $D^{n}(R)$. Hence the second statement follows from the first one. We prove the first one by induction on $n$. Since $D^{0}(R)$ is generated, as algebra, by $\left\{\lambda_{r}, \rho_{s} \mid r, s \in R\right\}$, we see that the result holds for $n=0$. Assuming that the result holds true for $n-1$, let $\lambda_{r} \varphi \in D^{n}(R)$ be such that $[\varphi, x] \in D^{n-1}(R)$ for all $x \in R$. For $x_{1}, x_{2} \in R$, we have

$$
\left[\left[\lambda_{r} \varphi, x_{1}\right], x_{2}\right]=\lambda_{\left[r, x_{1}\right]}\left[\varphi, x_{2}\right]+\lambda_{\left[r, x_{2}\right]}\left[\varphi, x_{1}\right]+\lambda_{r}\left[\left[\varphi, x_{1}\right], x_{2}\right]
$$

which is in $D^{n-1}(R)$. Hence the proposition.

Corollary 4.0.2. Let $R$ be a supercommutative superalgebra. Then

$$
D_{\beta}(R) \subset D(R) \subset D_{q}(R) .
$$

Proof. The proof of Proposition 3.0.1 shows that $D_{\beta}^{0}(R) \subset D(R)$. Assume that $D_{\beta}^{n}(R) \subset$ $D(R)$. Let $\varphi \in D_{\beta}^{n+1}(R)$ be homogeneous. Then, by Proposition 3.0.1, $[\varphi, a]_{\beta} \in D_{\beta}^{n}(R) \subset$ $D(R)$. If $\varphi$ is an even morphism (that is, $p(\varphi)=0$ ), then $[\varphi, a]=[\varphi, a]_{\beta} \in D^{n}(R)$ for all $a \in R$, hence $\varphi \in D(R)$. If $\varphi$ is an odd homomorphism and $a$ homogeneous, then

$$
[\varphi, a]=\left((-1)^{p(a)}-1\right) a \varphi+[\varphi, a]_{\beta}
$$

which, in turn, is in $D(R)$ since $a \varphi$ is even when $p(a)=1$. Hence the first inclusion. The second inclusion follows from Remark 2.3.1 and the definition of $D_{q}(R)$.

Remark 4.0.3. The inclusions in the corollary need not be filtration preserving. In general, these inclusions can be strict inclusions. For instance, for odd $r \in R$, the operator $\rho_{r}$ lies in $D(R)$ but need not lie in $D_{\beta}(R)$, and $\sigma_{1}$ lies in $D_{q}(R)$ but need not be in $D(R)$ if $R$ is not finitely generated.

\subsection{A special case}

Let $R=\mathbb{k}[\mathbf{X} ; n] \otimes \Lambda[\mathbf{Y} ; m]$ be a supercommutative superalgebra defined by $p\left(x_{i}\right)=0$ and $p\left(y_{i}\right)=1$.

Let us compute $D_{\beta}(R), D_{q}(R)$ and $D(R)$. Since $\mathbb{k}[\mathbf{X} ; n]$ is trivially graded,

$$
D_{\beta}(\mathbb{k}[\mathbf{X} ; n])=D_{q}(\mathbb{k}[\mathbf{X} ; n])=D(\mathbb{k}[\mathbf{X} ; n]) .
$$


Note, $D(\mathbb{k}[\mathbf{X} ; n])$ is the Weyl algebra for chark $=0$. By Remarks 2.1.1, 2.2.1 and 2.3.1, it suffices to compute $D_{\beta}(\Lambda[\mathbf{Y} ; m]), D_{q}(\Lambda[\mathbf{Y} ; m])$ and $D(\Lambda[\mathbf{Y} ; m])$. We already know that

$$
D_{\beta}(\Lambda[\mathbf{Y} ; m]) \subset D(\Lambda[\mathbf{Y} ; m]) \subset D_{q}(\Lambda[\mathbf{Y} ; m]) .
$$

For each $i \in\{1,2, \cdots, m\}$, we have $\lambda_{y_{i}}, \partial_{i} \in D_{\beta}(\Lambda[\mathbf{Y} ; m])$, where $\partial_{i}\left(y_{j}\right)=\delta_{i j}$; extend $\partial_{i}$ as a superderivation. It is easy to show that the $\mathbb{k}$-algebra generated by $\lambda_{y_{i}}$ and $\partial_{i}$ generates $\operatorname{End}_{\mathbb{k}}(\Lambda[\mathbf{Y} ; m])$ (the proof requires $m$ to be finite). Hence,

$$
D_{\beta}(R)=D(R)=D_{q}(R)=D(\mathbb{k}[\mathbf{X} ; n]) \otimes \operatorname{End}_{\mathbb{k}}(\Lambda[\mathbf{Y} ; m]) .
$$

More is true.

Proposition 4.1.1. Let $R$ be a supercommutative superalgebra such that the number of odd generators is finite. Then

$$
D_{\beta}(R)=D(R)=D_{q}(R) .
$$

Proof. Let $\left\{a_{1}, a_{2}, \cdots, a_{m}\right\}$ be the set of odd generators of $R$. By induction on $m$ we show that $D_{q}^{m}(R) \subset D_{\beta}(R)$.

Note that $\rho_{r}=\lambda_{r}$ for $r$ even and $\rho_{r}=\lambda_{r} \sigma_{1}$ for $r$ odd. Since $D_{\beta}^{0}(R)=\left\{\lambda_{r} \mid r \in R\right\}$, it suffices to show that $\sigma_{1} \in D_{\beta}(R)$. Since $\sigma_{1}$ is an even homomorphism, $\left[\sigma_{1}, r\right]_{\beta}=\left[\sigma_{1}, r\right]$. For $r$ even, $\left[\sigma_{1}, r\right]_{\beta}=0$. For $r$ odd, say $r=a_{1}$, we have $\left[\sigma_{1}, a_{1}\right]_{\beta}=-2 a_{1} \sigma_{1}$. Further, for $r$ even, we see that $\left[a_{1} \sigma_{1}, r\right]_{\beta}=0$ and for $r$ odd, say $r=a_{2}$, we have $\left[a_{1} \sigma_{1}, a_{2}\right]_{\beta}=-2 a_{1} a_{2} \sigma_{1}$. Proceeding similarly, we see that

$$
\left[\cdots\left[\left[\sigma_{1}, a_{1}\right]_{\beta}, a_{2}\right]_{\beta}, \cdots a_{n}\right]_{\beta}=(-2)^{n} a_{1} a_{2} \cdots a_{n} \sigma_{1} .
$$

From this it follows that $\sigma_{1} \in D_{\beta}^{n+1}(R)$.

Assume that $D_{q}^{m}(R) \subset D_{\beta}(R)$. By Remark 2.2.1, we need to consider $\varphi \in D_{q}^{m+1}(R)$ such that

$$
[\varphi, r] \in D_{q}^{m}(R) \text { for all } r \in R .
$$

If $\varphi$ is an even homomorphism, then $[\varphi, r]_{\beta}=[\varphi, r] \in D_{q}^{m}(R)$, and hence $\varphi \in D_{\beta}(R)$. If $\varphi$ is an odd homomorphism, then consider

$$
\left[\varphi, a_{1}\right]_{\beta}=\varphi a_{1}+a_{1} \varphi=\left[\varphi, a_{1}\right]+2 a_{1} \varphi
$$

which is an even homomorphism with $\left[\varphi, a_{1}\right] \in D_{\beta}(R)$. We next see that

$$
\left[\left[\varphi, a_{1}\right]_{\beta}, a_{2}\right]_{\beta}=4 a_{1} a_{2} \varphi+\text { something in } D_{\beta}(R) .
$$

Proceeding thus, we have

$$
\left[\cdots\left[\left[\varphi, a_{1}\right]_{\beta}, a_{2}\right]_{\beta}, \cdots a_{n}\right]_{\beta}=2^{n} a_{1} a_{2} \cdots a_{n} \varphi+\text { something in } D_{\beta}(R) .
$$

One further $\beta$-commutator places $\varphi$ in $D_{\beta}(R)$. Hence the proposition. 
Let $R, S$ be two superalgebras. Then $R \stackrel{s}{\otimes} S$ is a superalgebra whose superspace is $R \otimes S$ with the usual addition, and the multiplication given by

$$
(a \otimes b)(c \otimes d)=(-1)^{p(b) p(c)}(a c \otimes b d) .
$$

If $R$ and $S$ are supercommutative, then $R \stackrel{s}{\otimes} S$ is also supercommutative.

The natural maps

$$
\begin{aligned}
& \operatorname{Hom}(R, R) \longrightarrow \operatorname{Hom}(R \stackrel{s}{\otimes} S, R \stackrel{s}{\otimes} S), \varphi \mapsto(r \otimes s \mapsto \varphi(r) \otimes s) \\
& \operatorname{Hom}(S, S) \longrightarrow \operatorname{Hom}(R \stackrel{s}{\otimes} S, R \stackrel{s}{\otimes} S), \quad \psi \mapsto\left(r \otimes s \mapsto r \otimes \psi_{0}(s)+\sigma_{1}(r) \otimes \psi_{1}(s)\right),
\end{aligned}
$$

where $\psi_{0}$ is the even part and $\psi_{1}$ is the odd part of $\psi$, give rise to a map

$$
\operatorname{Hom}(R, R) \stackrel{s}{\otimes} \operatorname{Hom}(S, S) \longrightarrow \operatorname{Hom}(R \stackrel{s}{\otimes} S, R \stackrel{s}{\otimes} S) .
$$

Note that the tensor product on the left is the tensor product of superalgebras, which is ensured because of the altered action (4.1.3) of $\operatorname{Hom}(S, S)$ on $R \stackrel{s}{\otimes} S$ above.

Remark 4.1.1. If $d_{R}, d_{S}$ are superderivations of superalgebras $R, S$ respectively, then the morphism $d_{R \otimes S} \in \operatorname{Hom}(R \stackrel{s}{\otimes} S, R \stackrel{s}{\otimes} S)$ defined by

$$
d_{R \otimes S}(r \otimes s)=d_{R}(r) \otimes s+r \otimes\left(d_{S}\right)_{0}(s)+\sigma_{1}(r) \otimes\left(d_{S}\right)_{1}(s)
$$

gives a superderivation of $R \stackrel{s}{\otimes} S$.

The following proposition is similar to Theorem 3.1.1 of [4]. We present it here for completeness of discussion.

Proposition 4.1.2. If $R, S$ are finitely generated by homogeneous elements, then there is an isomorphism of filtered superalgebras

$$
D_{\beta}(R) \stackrel{s}{\otimes} D_{\beta}(S) \cong D_{\beta}(R \stackrel{s}{\otimes} S) .
$$

Proof. For any $\varphi \in D_{\beta}(R)$ and $\psi \in D_{\beta}(S)$, we have

$$
[\varphi \otimes \psi, r \otimes s]_{\beta}=(-1)^{p(\psi) p(r)}\left([\varphi, r]_{\beta} \otimes \psi s+(-1)^{p(\varphi) p(r)} r \varphi \otimes[\psi, s]_{\beta}\right) .
$$

Hence, $D_{\beta}^{a}(R) \stackrel{s}{\otimes} D_{\beta}^{b}(S) \subset D_{\beta}^{a+b}(R \stackrel{s}{\otimes} S)$.

It remains to show that if a homogeneous $\varphi \in \operatorname{Hom}(R \stackrel{s}{\otimes} S)$ is such that $[\varphi, r \otimes s]_{\beta} \in$ $D_{\beta}(R) \stackrel{s}{\otimes} D_{\beta}(S)$ for all $r \otimes s \in R \stackrel{s}{\otimes} S$, then $\varphi \in D_{\beta}(R) \stackrel{s}{\otimes} D_{\beta}(S)$.

By changing $\varphi$ to $\varphi-\lambda_{\varphi(1 \otimes 1)}$, we assume that $\varphi(1 \otimes 1)=0$.

First consider the case where $[\varphi, r \otimes 1]_{\beta}=0$ for all $r \in R$. Let $\left\{s_{1}, \cdots, s_{n}\right\}$ be a finite set of homogeneous generators of $S$ over $\mathbb{k}$. Let $\eta_{i}$ denote $\left[\varphi, 1 \otimes s_{i}\right]_{\beta}$. For each $i$, let $\eta_{i}=\sum \eta_{i(1)} \otimes \eta_{i(2)} \in D_{\beta}(R) \stackrel{s}{\otimes} D_{\beta}(S)$, where $\eta_{i(1)}$ and $\eta_{i(2)}$ are homogeneous. 
Note that for homogeneous $r$,

$\left[\left[\varphi, 1 \otimes s_{i}\right]_{\beta}, r \otimes 1\right]_{\beta}=(-1)^{p\left(s_{i}\right) p(r)}\left[[\varphi, r \otimes 1]_{\beta}, 1 \otimes s_{i}\right]_{\beta}=0$.

So, we may assume that $\left[\eta_{i(1)}, r\right]_{\beta}=0$ for all $r \in R$.

Let $V_{1} \subset D_{\beta}(R)$ be the finite dimensional super vector space spanned by the $\eta_{i(1)}$. Every element of $V_{1}$ commutes with $r \in R$. For $s, s^{\prime} \in S$, we have

$$
\left[\varphi, 1 \otimes s s^{\prime}\right]_{\beta}=[\varphi, 1 \otimes s]_{\beta}\left(1 \otimes s^{\prime}\right)+(-1)^{p(\varphi) p(s)}(1 \otimes s)\left[\varphi, 1 \otimes s^{\prime}\right]_{\beta} .
$$

Hence, every $[\varphi, 1 \otimes s]_{\beta}$ can be expressed as $\sum_{i=1}^{l} f_{i} \otimes g_{i}^{s}$, where $\left\{f_{i}\right\}_{i=1}^{l} \subset D_{\beta}(R)$ is a homogeneous basis of $V_{1}$, and $g_{i}^{s} \in D_{\beta}(S)$, where $p\left(g_{i}^{s}\right)=p(\varphi)+p(s)+p\left(f_{i}\right)$. For each $i=1, \cdots, l$, define a function $h_{i}: S \longrightarrow S$ setting $h_{i}(s)=g_{i}^{s}(1)$. Since $[\varphi, 1 \otimes s]_{\beta}$ is linear in $s$, it follows that $h_{i} \in \operatorname{Hom}(S, S)$. Also, $p\left(h_{i}\right)=p\left(f_{i}\right)+p(\varphi)$, and hence is independent of $s$.

For $s, t \in S$, consider

$$
\left[h_{i}, s\right]_{\beta}(t)=h_{i}(s t)-(-1)^{p\left(h_{i}\right) p(s)} s h_{i}(t)=g_{i}^{s t}(1)-(-1)^{p\left(h_{i}\right) p(s)} s g_{i}^{t}(1) .
$$

Since

$$
[\varphi, 1 \otimes s t]_{\beta}=[\varphi, 1 \otimes s]_{\beta}(1 \otimes t)+(-1)^{p(\varphi) p(s)}(1 \otimes s)[\varphi, 1 \otimes t]_{\beta},
$$

we have

$$
\begin{aligned}
\sum_{i} f_{i} \otimes g_{i}^{s t} & =\left(\sum_{i} f_{i} \otimes g_{i}^{s}\right)(1 \otimes t)+(-1)^{p(\varphi) p(s)}(1 \otimes s) \sum_{i} f_{i} \otimes g_{i}^{t} \\
& =\sum_{i} f_{i} \otimes g_{i}^{s} t+(-1)^{p(\varphi) p(s)+p\left(f_{i}\right) p(s)} \sum_{i} f_{i} \otimes s g_{i}^{t} \\
& =\sum_{i} f_{i} \otimes g_{i}^{s} t+(-1)^{\left(p(\varphi)+p\left(f_{i}\right)\right) p(s)} \sum_{i} f_{i} \otimes s g_{i}^{t} .
\end{aligned}
$$

That is, $g_{i}^{s t}=g_{i}^{s} t+(-1)^{\left(p(\varphi)+p\left(f_{i}\right)\right) p(s)} s g_{i}^{t}$. Thus, $\left[h_{i}, s\right]_{\beta}(t)=g_{i}^{s} \in D_{\beta}(S)$ which further implies that $h_{i} \in D_{\beta}(S)$. Hence, $\psi=\sum f_{i} \otimes h_{i} \in D_{\beta}(R) \stackrel{s}{\otimes} D_{\beta}(S)$. One can see that $[\psi, r \otimes 1]_{\beta}=[\varphi, r \otimes 1]_{\beta}=0$ and $[\psi, 1 \otimes s]_{\beta}=[\varphi, 1 \otimes s]_{\beta}$. Since $\psi(1 \otimes 1)=\varphi(1 \otimes 1)=0$, we have $\varphi=\psi \in D_{\beta}(R) \stackrel{s}{\otimes} D_{\beta}(S)$.

Now consider any $\varphi \in D_{\beta}(R \stackrel{s}{\otimes} S)$ such that $[\varphi, r \otimes s]_{\beta} \in D_{\beta}(R) \stackrel{s}{\otimes} D_{\beta}(S)$ with $\varphi(1 \otimes 1)=0$. Let $\left\{r_{1}, r_{2}, \cdots, r_{m}\right\}$ be a set of homogeneous generators of $R$. Let

$$
\left[\varphi, r_{i} \otimes 1\right]=\mu_{i}=\sum \mu_{i(1)} \otimes \mu_{i(2)} \in D_{\beta}(R) \stackrel{s}{\otimes} D_{\beta}(S) .
$$

Let $V_{2} \subset D_{\beta}(S)$ denote the finite dimensional super vector space spanned by the $\mu_{i(2)}$. Let $\left\{g_{1}, g_{2}, \cdots, g_{p}\right\}$ be a basis of homogeneous elements in $V_{2}$. Let $[\varphi, r \otimes 1]=\sum_{i} f_{i}^{r} \otimes g_{i}$ for $f_{i}^{r} \in D_{\beta}(R)$. For each $i$, let $e_{i} \in \operatorname{Hom}(R, R)$ denote the homogeneous homomorphism given by $e_{i}(r)=f_{i}^{r}(1)$. As before, we can see that $\left[e_{i}, r\right]_{\beta}=f_{i}^{r} \in D_{\beta}(R)$. Hence, $\psi=\sum e_{i} \otimes g_{i} \in D_{\beta}(R) \stackrel{s}{\otimes} D_{\beta}(S)$. We have,

$$
(\varphi-\psi)(1 \otimes 1)=0, \quad[(\varphi-\psi), r \otimes 1]_{\beta}=0, \text { and }[(\varphi-\psi), r \otimes s]_{\beta} \in D_{\beta}(R) \stackrel{s}{\otimes} D_{\beta}(S) .
$$

As before, $\varphi-\psi \in D_{\beta}(R) \stackrel{s}{\otimes} D_{\beta}(S)$ which proves the result. 


\subsubsection{The universal supercommutative envelope}

Every metaabelian algebra is a nonhomogeneous subalgebra of a supercommutative superalgebra. Its universal supercommutative envelope was constructed by Volichenko as follows [8].

For a metaabelian $M$, let $M^{(+)}$denote the related Jordan algebra. That is, as a set $M^{(+)}=\left\{m^{(+)} \mid m \in M\right\}$. The vector space structure on $M^{(+)}$is given by $\left(m^{(+)}+n^{(+)}\right)=$ $(m+n)^{(+)}$and $\alpha m^{(+)}=(\alpha m)^{(+)}$for any $\alpha \in \mathbb{k}$ and $m, n \in M$. The multiplication on $M^{(+)}$is defined as

$$
m^{(+)} \circ n^{(+)}=\frac{1}{2}(m n+n m)^{(+)} .
$$

Let $C$ denote the subalgebra of $M$ generated by the elements $[m, n]$ for any $m, n \in M$. Let $\Omega_{M^{(+)} / C}^{1}$ denote the $M^{(+)}$-module of differentials, i.e., $\Omega_{M^{(+)} / C}^{1}$ is the quotient of the free $M^{(+)}$-module with basis $d m$ where $m \in M$, modulo the submodule generated by

$$
d(m+n)-d m-d n, \quad d(m n)-m^{(+)} d n-n^{(+)} d m, \text { for any } m, n \in M .
$$

Note that $d c=0$ for any $c \in C$ in $\Omega_{M^{(+)} / C}^{1}$. The universal supercommutative envelope of $M$ is $S_{M}=M^{(+)} \oplus \Omega_{M^{(+)} / C}^{1}$, whose even component is $M^{(+)}$and the odd component is $\Omega_{M^{(+)} / C}^{1}$; the product of odd elements being $d m \cdot d n:=\frac{1}{2}[m, n]^{(+)}$. The metaabelian algebra $M$ can be seen as a nonhomogeneous subspace $\left\{m^{(+)}+d m \mid m \in M\right\}$ of $S_{M}$.

\subsubsection{Tensor products of metaabelian algebras}

A special tensor product of metaabelian algebras was constructed by V Molotkov in an Appendix to [8]. Here we present another, simpler, construction which also satisfies the universal property of Proposition 4.1.3.

We first describe metaabelian tensor product of two (possibly nonhomogeneous) subalgebras of superalgebras.

Let $S$ and $T$ be two $\mathbb{k}$-superalgebras. Let $A \subset S$ and $B \subset T$ be subalgebras, possibly nonhomogeneous. We have an inclusion of vector spaces $A \otimes B \subset S \stackrel{S}{\otimes} T$. The multiplication (4.1.1) on $S \stackrel{s}{\otimes} T$ with a natural parity makes $S \stackrel{s}{\otimes} T$ into a superalgebra. The vector space $A \otimes B$ is not necessarily closed under this multiplication. For instance

$$
(1 \otimes b)(a \otimes 1)=\left(a \otimes b-2 a_{1} \otimes b_{1}\right) \notin A \otimes B,
$$

where $a_{1}, b_{1}$ are the odd parts of $a, b$, respectively, for $a \in A$ and $b \in B$. Therefore, define the metaabelian tensor product of algebras $A$ and $B$ to be the subalgebra of $S \stackrel{s}{\otimes} T$ generated by $A \otimes B$ and denote it by $A \stackrel{m}{\otimes} B$.

There are natural maps of algebras $i_{A}: A \longrightarrow A \stackrel{m}{\otimes} B$ and $i_{B}: B \longrightarrow A \stackrel{m}{\otimes} B$ given by $i_{A}(a)=a \otimes 1$ and $i_{B}(b)=1 \otimes b$.

Remark 4.1.2. The metaabelian tensor product is dependent on its ambient space. For instance, consider the following two examples:

(1) Let $\mathbb{k}[x]$ and $\mathbb{k}[y]$ be purely even. Then, $\mathbb{k}[x] \otimes \mathbb{k}[y]=\mathbb{k}[x, y]$. 
(2) Let $\mathbb{k}\left[z_{1}\right]=\mathbb{k}\left[x_{0}+x_{1}\right]$ and $\mathbb{k}\left[z_{2}\right]=\mathbb{k}\left[y_{0}+y_{1}\right]$ be metaabelian algebras in their supercommutative envelopes $\mathbb{k}\left[x_{0}\right] \otimes \Lambda\left(x_{1}\right)$ and $\mathbb{k}\left[y_{0}\right] \otimes \Lambda\left(y_{1}\right)$. Then,

$$
\mathbb{k}\left[z_{1}, z_{2}\right]:=\mathbb{k}\left[z_{1}\right] \stackrel{m}{\otimes} \mathbb{k}\left[z_{2}\right]=\mathbb{k}\left[x_{0}+x_{1}\right] \stackrel{m}{\otimes} \mathbb{k}\left[y_{0}+y_{1}\right]=\mathbb{k}\left[x_{0}+x_{1}, y_{0}+y_{1}\right]
$$

is a metaabelian subalgebra in $\mathbb{k}\left[x_{0}, y_{0}\right] \otimes \Lambda\left(x_{0}, y_{0}\right)$. Observe that $\mathbb{k}\left[z_{1}, z_{2}\right]$ is no longer commutative. Hence, it is not algebraically isomorphic to $\mathbb{k}[x, y]$ even though $\mathbb{k}[x] \cong \mathbb{k}\left[z_{1}\right]$ and $\mathbb{k}[y] \cong \mathbb{k}\left[z_{2}\right]$ as algebras.

Let $M$ and $N$ be metaabelian algebras. Let $S_{M}$ and $S_{N}$ be their supercommutative envelopes.

The supercommutative envelopes are universal constructions. So, we have natural inclusions $M \subset S_{M}$ and $N \subset S_{N}$. By construction $M \stackrel{m}{\otimes} N$ is metaabelian. This product possesses universal properties:

Proposition 4.1.3. Let $A, B, C$ be three metaabelian algebras. Let $f: A \longrightarrow C$ and $g: B \longrightarrow C$ be algebra homomorphisms. Then there exists a unique algebra homomorphism $h: A \stackrel{m}{\otimes} B \longrightarrow C$ such that $f=h \circ i_{A}$ and $g=h \circ i_{B}$.

Proof. An algebra homomorphism $f: A \longrightarrow C$ naturally gives an even algebra homomorphism of supercommutative superalgebras $S_{f}: S_{A} \longrightarrow S_{C}$ such that the restriction of $S_{f}$ on $A$ is $f$. Likewise, we have an even algebra homomorphism of supercommutative superalgebras $S_{g}: S_{B} \longrightarrow S_{C}$ such that the restriction of $S_{g}$ on $B$ is $g$.

Since $S_{A} \stackrel{s}{\otimes} S_{B}$ is a tensor product with universal properties, we have the unique map $S_{h}: S_{A} \stackrel{s}{\otimes} S_{B} \longrightarrow S_{C}$. By construction, $S_{h}(a \otimes 1)=S_{f}(a)=f(a) \in C$ and $S_{h}(1 \otimes b)=S_{g}(b)=g(b) \in C$ for any $a \in A$ and $b \in B$. Denote by $h$ the restriction of $S_{h}$ on $A \stackrel{m}{\otimes} B$. Then we have $f=i_{A} \circ h$ and $g=i_{B} \circ h$.

Let a map $l: A \stackrel{m}{\otimes} B \longrightarrow C$ be given such that $f=l \circ i_{A}$ and $g=l \circ i_{B}$. Then, we have $h(a \otimes 1)=S_{f}(a \otimes 1)=l(a \otimes 1)$ and $h(1 \otimes b)=S_{g}(1 \otimes b)=l(1 \otimes b)$. Hence, $h=l$.

Proposition 4.1.4. Let $M, N$ be two metaabelian algebras, $S_{M}, S_{N}$ their corresponding supercommutative envelopes. Then $S_{M} \stackrel{s}{\otimes} S_{N}$ is the supercommutative envelope of $M \stackrel{m}{\otimes} N$.

Proof. We write $S_{M}=M^{(+)} \oplus \Omega_{M^{(+)} / C_{M}}^{1}$ and $S_{N}=N^{(+)} \oplus \Omega_{N^{(+)} / C_{N}}^{1}$, where $C_{M}$ and $C_{N}$ are the commutator subalgebras of $M$ and $N$ respectively. We need to show that

- $(M \stackrel{m}{\otimes} N)^{(+)}=\left(S_{M} \stackrel{s}{\otimes} S_{N}\right)_{0} ;$

- $\left(S_{M} \stackrel{s}{\otimes} S_{N}\right)_{1}=\Omega_{(M \otimes N)^{(+)} / C_{M \otimes N}^{m}}^{1}$ is the module of differentials over $(M \stackrel{m}{\otimes} N)^{(+)}$ generated by $d_{S_{M} \otimes S_{N}} r$ for $r \in(M \stackrel{m}{\otimes} N)$;

- $C_{M \otimes N}^{m} \subset \operatorname{Ker}\left(d_{S_{M} \otimes S_{N}}\right)$, 
where $d_{S_{M} \otimes S_{N}}$ is the derivation of $S_{M} \stackrel{s}{\otimes} S_{N}$ defined in Remark (4.1.1). Note that $d_{S_{M} \otimes S_{N}}$ is odd and $d_{S_{M} \otimes S_{N}}^{2}=0$. We will use the letter $d$ to mean any of the derivations $d_{M}, d_{N}, d_{S_{M} \otimes S_{N}}$ as will be clear from the context. We have an isomorphism

$$
\begin{aligned}
(M \stackrel{m}{\otimes} N)^{(+)} & \longrightarrow\left(S_{M} \stackrel{s}{\otimes} S_{N}\right)_{0} \\
(m \otimes n)^{(+)} & \mapsto\left(m^{(+)} \otimes n^{(+)}\right)+(d m \otimes d n)
\end{aligned}
$$

for any $m \in M, n \in N$. Similarly, note that

$$
d(m \otimes n)=d m \otimes n^{(+)}+m^{(+)} \otimes d n .
$$

Hence $\left(S_{M} \stackrel{s}{\otimes} S_{N}\right)_{1}$ is generated over $(M \stackrel{m}{\otimes} N)^{(+)}$by $d r$ for $r \in(M \stackrel{m}{\otimes} N)$. Lastly, we see that $C_{M \otimes N}$ is generated by elements of the kind $e[r, s]$ for $r, s \in(M \stackrel{m}{\otimes} N)$ and $e$ an even element, multiple of terms of the form $d m \otimes d n$ for $m \in M$ and $n \in N$. This implies that $d(e)=0$. So, it remains to show that $d([r, s])=0$ for and $r=(m \otimes n)$ and $s=(a \otimes b)$ where $m, a \in M$ and $n, b \in N$; this follows from a straightforward calculation.

Remark 4.1.3. Let $S=\mathbb{k}[\mathbf{X} ; n] \otimes \Lambda[\mathbf{Y} ; n]$ and $T=\mathbb{k}[\mathbf{A} ; m] \otimes \Lambda[\mathbf{B} ; m]$. Let $\mathbb{k}[Z]$ be the non-graded subalgebra in $S$ generated by all the elements $z_{i}=x_{i}+y_{i}$ and $\mathbb{k}[C]$ be the non-graded subalgebra in $T$ generated by all the elements $c_{i}=a_{i}+b_{i}$. Then, the tensor product of the two metaabelian algebras $\mathbb{k}[Z]$ and $\mathbb{k}[C]$ is the metaabelian algebra $\mathbb{k}[Z, C]$ contained in $\mathbb{k}[\mathbf{X}, \mathbf{A} ; n+m] \otimes \Lambda[\mathbf{Y}, \mathbf{B} ; n+m]$.

\subsection{Volichenko algebras as algebras of differential operators}

Volichenko algebras are to Lie superalgebras what metaabelian algebras are to supercommutative superalgebras ([8]): a Volichenko algebra is defined to be a non-homogeneous subspace of a Lie superalgebra closed under the superbracket. No intrinsic definition of Volichenko algebras (in terms of defining identities) is available yet, see [1], [8].

Recall that the algebra of usual differential operators on $M$ is

$$
\left.D(M):=\left\{\varphi \in \operatorname{End}_{\mathbb{k}}(M) \mid \cdots\left[\left[\varphi, m_{1}\right], m_{2}\right], \cdots m_{n}\right]=0 \text { for some } n \geq 0 \text { and any } m_{i} \in M\right\} .
$$

We also introduce other algebras of differential operators on $M, D_{\beta}(M)$ and $V D(M)$.

\subsection{1 $\beta$-differential operators on $M$}

We define the algebra of $\beta$-differential operators on $M$ as

$$
D_{\beta}(M)=D_{\beta}(S) \cap \operatorname{End}_{\mathbb{k}}(M)=\left\{\varphi \in D_{\beta}(S) \mid \varphi(M) \subset M\right\} .
$$

Remark 4.2.1. The following are immediate:

(1) $D_{\beta}(M) \subset D(M)$;

(2) $D_{\beta}(M)$ is a filtered algebra, $D_{\beta}(M)=\cup_{n} D_{\beta}^{n}(M)$, where

$$
D_{\beta}^{n}(M)=\left\{\varphi \in D_{\beta}^{n}(S) \mid \varphi(M) \subset M\right\} .
$$


For any $\varphi=\varphi_{0}+\varphi_{1} \in \operatorname{End}_{\mathbb{k}}(S)$ and $a=a_{0}+a_{1} \in S$, we let $\bar{a}=a_{0}-a_{1}$ and $\bar{\varphi}=\varphi_{0}-\varphi_{1}$. Consider the $\beta$-commutator,

$$
[\varphi, a]_{\beta}=\varphi a-\left(a_{0} \varphi+a_{1} \bar{\varphi}\right)=\varphi a-\left(a \varphi_{0}+\bar{a} \varphi_{1}\right) .
$$

It is not clear whether $[\varphi, a]_{\beta} \in D_{\beta}(M)$ if $\varphi \in D_{\beta}(M)$ and $a \in M$. The situation is remedied by introducing the Volichenko differential operators.

\subsubsection{Volichenko differential operators on $M$}

The construction of a supercommutative envelope of $M$ gives us a natural superderivation $d: S \longrightarrow S$ of parity 1 given on the generators by

$$
d\left(m^{(+)}\right)=d m \text { and } d(d m)=0 .
$$

Clearly, $d^{2}=0$. For an even $\varphi_{0} \in D_{\beta}^{n}(S)$, one can show by induction on $n$ that $\left[d, \varphi_{0}\right] \in$ $D_{\beta}^{n}(S)$. Define the algebra of Volichenko differential operators as

$$
V D(M)=\left\{\varphi_{0}+\left[d, \varphi_{0}\right] \mid \varphi_{0} \in D_{\beta}(S) \text { is even }\right\} .
$$

The following proposition justifies the terminology used above.

Proposition 4.2.1. (1) For any even $\varphi_{0} \in D_{\beta}(S)$, we have $\left(\varphi_{0}+\left[d, \varphi_{0}\right]\right)(m) \in M$ for all $m \in M$. In particular, $V D(M) \subset D_{\beta}(M)$.

(2) The vector space $V D(M)$ is a filtered algebra, $V D(M)=\cup_{n \geq 0} V D^{n}(M)$, where

$$
V D^{n}(M)=\left\{\varphi_{0}+\left[d, \varphi_{0}\right] \mid \varphi_{0} \in D_{\beta}^{n}(S) \text { is even }\right\} .
$$

In particular, $V D(M) \subset D_{\beta}(M)$ as filtered algebras.

(3) $V D^{0}(M)=\left\{\lambda_{m} \mid m \in M\right\}$ and $V D^{1}(M)=V D^{0}(M) \oplus V$ as vector spaces where $V$ is a Volichenko algebra.

(4) For any $\varphi=\varphi_{0}+\left[d, \varphi_{0}\right] \in V D^{n}(M)$, we have $[\varphi, m]_{\beta} \in V D^{n-1}(M)$ for any $m \in M$. In particular, if $\varphi \in V D^{n}(M)$, then $\left.\left[\cdots\left[\varphi, m^{0}\right]_{\beta}, m^{1}\right]_{\beta}, \cdots, m^{n}\right]_{\beta}=0$, where $m^{i} \in M$.

Proof. (1) We write $m=m^{(+)}+d m$ and

$$
\left(\varphi_{0}+\left[d, \varphi_{0}\right]\right)(m)=\left(\varphi_{0}\left(m^{(+)}\right)+\left[d, \varphi_{0}\right](d m)\right)+\left(\varphi_{0}(d m)+\left[d, \varphi_{0}\right]\left(m^{(+)}\right)\right) .
$$

From direct checking it follows that

$$
d\left(\varphi_{0}\left(m^{(+)}\right)+\left[d, \varphi_{0}\right](d m)\right)=\left(\varphi_{0}(d m)+\left[d, \varphi_{0}\right]\left(m^{(+)}\right)\right) .
$$

(2) For $\varphi_{0}, \psi_{0} \in D_{\beta}(S)$ even, we see that $\left[d, \varphi_{0}\right]\left[d, \psi_{0}\right]$ is an even homomorphism and $\left[d,\left[d, \varphi_{0}\right]\left[d, \psi_{0}\right]\right]=0$. Thus, $\left[d, \varphi_{0}\right]\left[d, \psi_{0}\right] \in V D(M)$. Further

$$
\left(\varphi_{0}+\left[d, \varphi_{0}\right]\right)\left(\psi_{0}+\left[d, \psi_{0}\right]\right)=\left(\varphi_{0} \psi_{0}+\left[d, \varphi_{0} \psi_{0}\right]\right)+\left[d, \varphi_{0}\right]\left[d, \psi_{0}\right] \in V D(M) .
$$

Hence, $V D(M)$ is an algebra.

(3) The first part follows from $\left[d, \lambda_{m^{(+)}}\right]=\lambda_{d m}$. As spaces, $D_{\beta}^{1}(S)=D_{\beta}^{0}(S) \oplus \operatorname{Der}_{\beta}(S)$, where $\operatorname{Der}_{\beta}(S)$ is a Lie superalgebra (see Corollary 3.0.1). The odd derivation $d \in D_{\beta}^{1}(S)$ 
is homologic (i.e., $[d, d]_{\beta}=0$ ). Hence, by Example 2.5 of $[8], V D^{1}(M)$ is a Volichenko algebra.

(4) For $m=m_{0}+d m \in M$ and $\varphi=\varphi_{0}+\left[d, \varphi_{0}\right]$, we have

$$
\left.[\varphi, m]_{\beta}=\left[\varphi_{0}, m_{0}\right]+\left[d, \varphi_{0}\right] d m+d m\left[d, \varphi_{0}\right]\right]+\left[\varphi_{0}, d m\right]+\left[\left[d, \varphi_{0}\right], m_{0}\right] .
$$

It is easy to check that

$$
\left[d,\left[\varphi_{0}, m_{0}\right]+\left[d, \varphi_{0}\right] d m+d m\left[d, \varphi_{0}\right]\right]=\left[\varphi_{0}, d m\right]+\left[\left[d, \varphi_{0}\right], m_{0}\right] .
$$

Remark 4.2.2. The result (4) of the above proposition, is not an if and only if statement. That is, if $[\varphi, m] \in V D^{n-1}(M)$, then $\varphi$ need not be in $V D^{n}(M)$. For example, consider $S=\mathbb{k}[x] \otimes \Lambda(y)$ a supercommutative superalgebra with $p(x)=0$ and $p(y)=1$, and let $M=\mathbb{k}[x+y]$, be the polynomial ring sitting diagonally in $S$. Let $\psi_{1}=x \partial_{y}+y \partial_{x} \in$ $\operatorname{Der}_{\beta}(S)$. We see that $\left[\psi_{1}, m\right]_{\beta}=\lambda_{\psi_{1}(m)} \in V D^{0}(M)$ and $\psi$ keeps $M$ invariant, but $\psi_{1}$ is a nonzero purely odd operator. Hence $\psi_{1} \notin V D^{1}(M)$.

Theorem 4.2.1. (1) $V D\left(\prod_{\alpha} M_{\alpha}\right) \cong \prod_{\alpha} V D\left(M_{\alpha}\right)$ as filtered algebras, where $M_{\alpha}$ is a metaabelian algebra for each $\alpha$.

(2) There is an inclusion $V D(M) \stackrel{m}{\otimes} V D(N) \longrightarrow V D(M \stackrel{m}{\otimes} N)$ of filtered algebras, where $M, N$ are finitely generated metaabelian algebras.

Proof. (1) For each $\alpha$, let $S_{\alpha}$ denote the supercommutative envelope of $M_{\alpha}$ with $d_{\alpha}$ the associated superderivation. The direct product $\prod_{\alpha} S_{\alpha}$ along with the superderivation $\prod_{\alpha} d_{\alpha}$ is the supercommutative envelope of $\prod_{\alpha} M_{\alpha}$. The result follows from Lemma 3.0.1.

(2) Let $S_{M}, S_{N}$ be the supercommutative envelopes of $M, N$ respectively. Since $M$ and $N$ are finitely generated, $S_{M}, S_{N}$ are finitely generated. Let the correspondng natural derivations be denoted by $d_{M}$ and $d_{N}$ respectively. Consider their tensor derivation $d_{M \otimes N}$ : $S_{M} \stackrel{s}{\otimes} S_{N} \longrightarrow S_{M} \stackrel{s}{\otimes} S_{N}$ given by

$$
d_{M \otimes N}(a \otimes b)=d_{M}(a) \otimes b+(-1)^{p(a)} a \otimes d_{N}(b) .
$$

As in extensions (4.1.2) and (4.1.3), we extend operators in $V D(M)$ and $V D(N)$ to $\operatorname{Hom}(M \stackrel{m}{\otimes} N, M \stackrel{m}{\otimes} N)$. By the nature of the extension, one can see that these extended operators are in $V D(M \stackrel{m}{\otimes} N)$. Proposition 4.1.2 completes the proof.

\subsubsection{An example}

Let $\mathbb{k}[Z]$ be the nonhomogeneous subalgebra of $S=\mathbb{k}[\mathbf{X} ; n] \otimes \Lambda[\mathbf{Y} ; n]$ generated by variables $Z=\left(z_{1}, z_{2}, \cdots, z_{n}\right)$, with $z_{i}=x_{i}+y_{i}$.

Note that $D_{\beta}(S)=D_{\beta}(\mathbb{k}[\mathbf{X} ; n]) \otimes D_{\beta}(\Lambda[\mathbf{Y} ; n])$. The algebra of differential operators on $\mathbb{k}[\mathbf{X} ; n]$ is the Weyl algebra. That is,

$$
D_{\beta}(\mathbb{k}[\mathbf{X} ; n])=\mathbb{k}<x_{1}, x_{2}, \cdots, x_{n}, \partial_{x_{1}}, \partial_{x_{2}}, \cdots, \partial_{x_{n}}>/ \operatorname{Rel},
$$

where $R e l$ is the set of relations generated by $\left[\partial_{x_{i}}, x_{j}\right]=\delta_{i, j},\left[x_{i}, x_{j}\right]=0=\left[\partial_{x_{i}}, \partial_{x_{j}}\right]$. Likewise,

$$
D_{\beta}(\Lambda[\mathbf{Y} ; n])=\mathbb{k}<y_{1}, y_{2}, \cdots, y_{n}, \partial_{y_{1}}, \partial_{y_{2}}, \cdots, \partial_{y_{n}}>/ R e l^{\prime},
$$


where $R e l^{\prime}$ is the set of relations generated by $\left[\partial_{y_{i}}, y_{j}\right]_{\beta}=\delta_{i, j},\left[y_{i}, y_{j}\right]_{\beta}=0=\left[\partial_{y_{i}}, \partial_{y_{j}}\right]_{\beta}$. Here, the operators $\partial_{y_{i}}$ and $y_{i}=\lambda_{y_{i}}$ are odd operators. The operators $\partial_{x_{i}}$ (and $\partial_{y_{i}}$ ) is an even (respectively, odd) superderivation on $S$ satisfying $\partial_{y_{i}}\left(y_{j}\right)=\delta_{i j}, \partial_{y_{i}}\left(x_{j}\right)=0$ (respectively, $\partial_{y_{i}}\left(y_{j}\right)=\delta_{i j}, \partial_{y_{i}}\left(x_{j}\right)=0$ ).

Note that $S$ is the supercommutative envelope of $\mathbb{k}[Z]$, with the natural odd derivation $d=\sum_{i} y_{i} \partial_{x_{i}}$. Hence,

$$
V D^{n}(\mathbb{k}[Z])=\left\{\varphi_{0}+\left[d, \varphi_{0}\right] \mid \varphi_{0} \in D_{\beta}^{n}(S) \quad \text { is even }\right\} .
$$

The subalgebra $\left(D_{\beta}(S)\right)_{0}$ of even elements in $D_{\beta}(S)$ is generated by

$$
\left\{x_{i}, \partial_{x_{i}}\right\}_{i} \cup\left\{y_{i} y_{j}, \partial_{y_{i}} \partial_{y_{j}}\right\}_{i<j} \cup\left\{y_{i} \partial_{y_{j}}\right\}_{i, j} .
$$

The following formulae are easy to check:

$$
\begin{aligned}
& {\left[d, x_{i}\right]=y_{i}, \quad\left[d, \partial_{x_{i}}\right]=0, \quad\left[d, y_{i} y_{j}\right]=0} \\
& {\left[d, \partial_{y_{i}} \partial_{y_{j}}\right]=\partial_{x_{i}} \partial_{y_{j}}-\partial_{y_{i}} \partial_{x_{j}}, \quad\left[d, y_{i} \partial_{y_{j}}\right]=-y_{i} \partial_{x_{j}} .}
\end{aligned}
$$

Hence, the Volichenko differential operators on $\mathbb{k}[Z]$ are generated by the operators, for $1 \leq i, j \leq n$,

- $x_{i}+y_{i}$ (this operator acts as left-multiplication by $z_{i}$ on $\mathbb{k}[Z]$ ),

- $\partial_{x_{i}}$ (this operator acts as the derivation $\partial_{z_{i}}$ on $\mathbb{k}[Z]$ ),

- $\partial_{y_{i}} \partial_{y_{j}}+\partial_{x_{i}} \partial_{y_{j}}-\partial_{y_{i}} \partial_{x_{j}}$ (this is a second order Volichenko differential operator acting nontrivially),

- $y_{i} \partial_{y_{j}}-y_{i} \partial_{x_{j}}$ (this is called a ghost operator, since it is a non-zero operator whose action on $\mathbb{k}[Z]$ is 0$)$.

Acknowledgments. This paper was written when the author was visiting Max-Planck Institut für Mathematik, Bonn and Indiana University, Bloomington. She would like to thank these two departments for their excellent working conditions. The author would like to thank D Leites for his patient teaching and V Molotkov for correspondence.

\section{References}

[1] Baranov A A, Volichenko Algebras and nonhomogeneous subalgebras of Lie superalgebras, Siberian mathematical Journal 36 (1995), 859-868, (Eng. trans.), Sibirskii Mat. Z. 36 (1995), 998-1009.

[2] Deligne P, Etingof P, Freed D, Jeffrey L, Kazhdan D, Morgan J, David R, Morrison D, and Witten E, Quantum fields and strings: a course for mathematicians, Vol. 1, 2, Amererican Mathematical Society, Providence, 1999.

[3] Duplij S, Bagger J, Siegel W (eds.) Concise Encyclopedia of Supersymmetry and Noncommutative Structures in Mathematics and Physics, Kluwer, Dordreht, 2003. 
[4] Iyer U N, Differential Operators on Hopf algebras and some functorial properties, Manuscripta math. 109 (2002), 121-129.

[5] Iyer U N, McCune $\mathrm{T} \mathrm{C}$, Quantum differential operators on $\mathbb{k}[x]$, International Journal of Mathematics 13 (2002), 395-413.

[6] Kac V G, Lie Superalgebras, Advances in Math. 26 (1997), 8-96.

[7] Leites D, Spectra of graded-commutative rings, Uspehi Matem. Nauk 29 (1974), 157-158 (in Russian).

[8] Leites D, Serganova V, Symmetries wider than supersymmetry, in Noncommutative structures in Mathematics and Physics, Editors: Duplij S and Wess J, 2001, $13-30$.

[9] Lunts V A, Rosenberg A L, Differential operators on noncommutative rings, Selecta Math.(N.S) 3 (1997), 335-359.

[10] Manin Yu I, Gauge field theory and complex geometry, Second edition, SpringerVerlag, Berlin, 1997. 